\title{
NATURAL FAMILIES OF CURVES IN A GENERAL CURVED
}

\section{SPACE OF $N$-DIMENSIONS*}

BY

\author{
JOSEPH LIPKE
}

\section{Introduction.}

In a recent paper $\dagger$ Professor Kasner gave a complete geometric characterization of the families of curves (termed natural families) defined as the extremals connected with variation problems of the form

$$
\int F d s=\text { minimum }
$$

where $F$ is any point function and $d s$ is the element of arc in the space considered. Kasner considered a euclidean space of any dimensionality (the results admit of direct extension to all spaces of constant curvature), though, for simplicity, he wrote out the results for three dimensions only.

It is the purpose of the present paper to obtain the complete geometric characterization of such natural families of curves in a general curved space (space of variable curvature in the Riemannian sense $\ddagger$ of any dimensionality. Such a space need not necessarily be contained in a euclidean spaee of one higher dimension, but represents a variety in a space of some higher dimensionality. The results obtained contain all former results as special cases.

The importance (in one direction) of the problem is sufficiently pointed out by Darboux, $\S$ who devotes a chapter, entitled "Le problème général de la dynamique" to a discussion of the general aspects of the problem of least action, pointing out its physical significance and making some geometric deductions therefrom. We quote the general statement of the problem:

* The two-dimensional results of this paper were presented to the Society at the Princeton meeting, September 13, 1909; the general results, at the New York meeting, December 29, 1910.

$\dagger$ KASNER, Natural families of trajectories: conservative fields of force, these Transa c tions, vol. 10 (1909), pp. 201-219.

$\ddagger$ Riemann, Ueber die Hypothesen welche der Geometrie zu Grunde liegen.

8 Darboux, Leçons sur la theorie générale des surfaces, vol. 2, p. 480. For a discussion of the Principle of Least Action, see also: Thomson and TaIt, treatise on natural philosophy, 1903, vol. 1, §327; P. AppelL, Traité de mécanique rationelle, vol. 1, p. 530. 
"Etant données deux positions $\left(P_{0}\right)$ et $\left(P_{1}\right)$ du système mobile, imaginons tous les déplacements continus qui amènent le systéme de la première position à la seconde, les vitesses satisfaisant à chaque instant à l'équation des forces vives

$$
2 T=\sum_{i k} a_{i, k} q_{i}^{\prime} q_{k}^{\prime}=2(U+h) .
$$

Si l'on considère l'intégrale

$$
\int_{\left(P_{0}\right)}^{\left(P_{1}\right)} m v^{2} d t=\int_{\left(P_{0}\right)}^{\left(P_{1}\right)} \sqrt{2 U+2 h} \sqrt{\sum_{i k} a_{i k} d q_{i} d q_{k}}
$$

relative à chacun de ces déplacements, elle sera moindre pour le mouvement naturel que pour tous les autres déplacements."

Here, $U$ is the work function (negative potential) and $h$ is the constant of energy. The totality of motions for all initial conditions gives $\infty^{2 n-i}$ trajectories; for each value of $h$, we get a family of $\infty^{2(n-1)}$ trajectories, and such a family is evidently a natural family. The complete system of $\infty^{2 n-1}$ trajectories in a conservative field of force is thus composed of $\infty^{1}$ natural families.

Several other important geometric and physical investigations lead to variation problems of type (1). Kasner points out among these: 1) the brachistochrone problem in the case of conservative forces; 2) the determination of the forms of equilibrium of a homogeneous flexible inextensible string acted upon by conservative forces; 3 ) the paths of light in an isotropic medium in which the index of refraction varies from point to point; 4) the conformal representation of the geodesics of any space upon some variety in higher space; thus the determination of the geodesics in a space, $V$, where

leads to a minimizing of

$$
d s^{2}=\sum_{i k} \lambda a_{i k} d x_{i} d x_{k}
$$

$$
\int \sqrt{\sum_{i k} \lambda a_{i k} d x_{i} d x_{k}}=\int \sqrt{\lambda} \sqrt{\sum_{i k} a_{i k} d x_{i} d x_{k}} .
$$

Hence the representing curves in the space, $V^{\prime}$, where

form a natural family.

$$
d s^{2}=\sum_{i k} a_{i k} d x_{i} d x_{k}
$$

The results obtained ( $\$ 1-4)$ for the complete geometric characterization of natural families of curves in any curved space, $V_{n}$, are as follows. (A) The locus of the centers of geodesic curvature of the $\infty^{n-1}$ curves which pass through any point of $V_{n}$ is a euclidean space of $n-1$ dimensions $\left(S_{n-1}\right)$. (B) The osculating geodesic surfaces ( $V_{2}$ 's) at any point of $V_{n}$ form a bundle of surfaces, i. e. all contain a fixed direction (and hence the geodesic in that direction), which is normal to the $S_{n-1}$ of property $A$. (C) The $n$ directions at any point 
of $V_{n}$, in which, as a consequence of properties $A$ and $B$, the osculating geodesic circles (curves of constant geodesic curvature) hyperosculate the curves of the natural family, are mutually orthogonal.

The three properties $A, B$, and $C$ are shown to be necessary and sufficient for the complete characterization. We thus answer the question: Given a family of $\infty^{2(n-1)}$ curves in a general curved space of $n$-dimensions, when will they be the extremals of a variation problem of type (1)?

Of course, properties $A$ and $B$ alone define a much larger class of families of curves than the natural families. This wider class, in the case $n=2(\$ 5)$ also includes the system of $\infty^{2}$ isogonal trajectories of a given set of $\infty^{1}$ curves as a special type. It is shown that there exists a geodesic curvature transformation which interchanges the two types - isogonal trajectories and natural families.

We might state here, as is evident from the form of (1), that the only point transformations which convert every natural family into a natural family are the transformations of the conformal group. For, if an integral of the type (1) is to be converted into one of the same form, the transformation must convert the expression $F d s$ into one of the same form, say $F_{1} d s$; hence $d s$ must be transformed into a multiple of itself - which property is characteristic of conformal transformations.

\section{\$1. The equations of the natural family.}

In a general curved space, $V_{n}$, the element of arc length is given by

$$
d s^{2}=\sum_{i k} a_{i k} d x_{i} d x_{k}^{*}
$$

Consider the coördinates of a moving, point of any extremal expressed as functions of the arc length $s$, measured from a fixed point of the curve; we thus have

$$
\sum_{i k} a_{i k} x_{i}^{\prime} x_{k}^{\prime}=1 . \dagger
$$

Hence (1) takes the form

$$
\int_{8_{0}}^{s_{1}} F V \overline{\sum_{i k} a_{i k} d x_{i} d x_{k}}=\text { minimum. }
$$

The extremals connected with an integral of this type are found to be applying the ordinary method of variation -

$$
\frac{d}{d s} \sum_{i} F a_{i l} x_{i}^{\prime}=\frac{1}{2} \sum_{i k} \frac{\partial\left(F^{2} a_{i l}\right)}{\partial x_{l}} x_{i}^{\prime} x_{k}^{\prime} \quad(l=1,2, \cdots, n) ;
$$

* We write only $\sum_{i k}$ and understand that the summation is to be carried out from 1 to $n$. for each of the indicated subscripts, unless otherwise specified.

$\dagger$ Throughout the paper, primes refer to total derivatives with respect to $s$. 
and on expanding this, we have

$$
\begin{array}{r}
\sum_{i} a_{i l} x_{i}^{\prime \prime}+\sum_{\lambda \mu}\left[\frac{\partial a_{\lambda l}}{\partial x_{\mu}}-\frac{1}{2} \frac{\partial a_{\lambda \mu}}{\partial x_{l}}\right] x_{\lambda}^{\prime} x_{\mu}^{\prime}=\frac{1}{F} \sum_{\lambda \mu}\left(\frac{\partial F}{\partial x_{l}} a_{\lambda \mu}-\frac{\partial F}{\partial x_{\mu}} a_{\lambda l}\right) x_{\lambda}^{\prime} x_{\mu}^{\prime} \\
(l=1,2, \ldots, n) .
\end{array}
$$

We find it most convenient to introduce the Christoffel three-indices symbols of the first and second kind, * defined by

$$
\begin{aligned}
{\left[\begin{array}{c}
\lambda \mu \\
l
\end{array}\right] } & =\frac{1}{2}\left(\frac{\partial a_{\lambda l}}{\partial x_{\mu}}+\frac{\partial a_{\mu l}}{\partial x_{\lambda}}-\frac{\partial a_{\lambda \mu}}{\partial x_{l}}\right), \\
\left\{\begin{array}{c}
\lambda \mu \\
i
\end{array}\right\} & =\sum_{l} A_{i l}\left[\begin{array}{c}
\lambda \mu \\
l
\end{array}\right], \quad \text { hence } \quad\left[\begin{array}{c}
\lambda \mu \\
l
\end{array}\right]=\sum_{i} a_{i l}\left\{\begin{array}{c}
\lambda \mu \\
i
\end{array}\right\},
\end{aligned}
$$

where $A_{i l}$ designates the minor of $a_{i l}$ in the determinant $a=\left|a_{\lambda \mu}\right|$ divided by $a$ itself.

Making use of the relations (2) and (4), we may now write $\left(3^{\prime}\right)$ as

$$
\sum_{i} a_{i l} x_{i}^{\prime \prime}+\sum_{\lambda \mu}\left[\begin{array}{c}
\lambda \mu \\
l
\end{array}\right] x_{\lambda}^{\prime} x_{\mu}^{\prime}=\frac{1}{F}\left(\frac{\partial F}{\partial x_{l}}-\sum_{\lambda \mu} \frac{\partial F}{\partial x_{\mu}} a_{\lambda l} x_{\lambda}^{\prime} x_{\mu}^{\prime}\right) \quad(l=1,2, \ldots, n)
$$

Solving these equations for $x_{i}^{\prime \prime}$, we get finally

where

$$
x_{i}^{\prime \prime}+\sum_{\lambda \mu}\left\{\begin{array}{c}
\lambda \mu \\
i
\end{array}\right\} x_{\lambda}^{\prime} x_{\mu}^{\prime}=\sum_{l} \frac{\partial L}{\partial x_{l}}\left(A_{i l}-x_{i}^{\prime} x_{l}^{\prime}\right) \quad(i=1,2, \ldots, n),
$$

$$
L=\log F \text {. }
$$

Conversely, we may easily verify that along the curves (6), the arc length $s$ is the parameter, i. e.

$$
\sum_{i k} a_{i k} x_{i}^{\prime} x_{k}^{\prime}=1
$$

These $n$ equations (6) taken together with (2) are the differential equations of the extremals connected with an integral of type $\left(1^{\prime}\right)$. Thus, any natural family of curves in $V_{n}$ is represented by differential equations of the form (6), where $L$ is an arbitrary point function.

From these equations it is evident that, given a point $x^{(0)}$ of $V_{n}$ and a direction $\xi \dagger$ through this point, one and only one curve of a given natural family of curves is determined. There are thus $\infty^{n-1}$ curves passing through a point and a totality of $\infty^{2(n-1)}$ curves composing the family.

\footnotetext{
* BinNCHI, Geometria differenziale, vol. 1, chap. II.

$\dagger$ Throughout, we shall use the symbol $x$, standing alone, for the set $x_{1}, x_{2}, \ldots, x_{n}$; and similarly $\xi$ to represent the set $\xi_{1}, \xi_{2}, \ldots, \xi_{n}$.
} 
§2. Centers of geodesic curvature; osculating geodesic surfaces.-Properties $A$ and $B$.

We shall first consider the radius of geodesic curvature* for any curve of the natural family. Combining the expression

$$
\frac{1}{\rho^{2}}=\sum_{i k} a_{i k}\left[x_{i}^{\prime \prime}+\sum_{\lambda \mu}\left\{\begin{array}{c}
\lambda \mu \\
i
\end{array}\right\} x_{\lambda}^{\prime} x_{\mu}^{\prime}\right]\left[x_{k}^{\prime \prime}+\sum_{\lambda \mu}\left\{\begin{array}{c}
\lambda \mu \\
k
\end{array}\right\} x_{\lambda}^{\prime} x_{\mu}^{\prime}\right] \dagger
$$

with the equations (6) of the natural family, we have

(8) $\frac{1}{\rho^{2}}=\sum_{i k} a_{i k}\left[\sum_{l m} \frac{\partial L}{\partial x_{l}} \frac{\partial L}{\partial x_{m}}\left(A_{i l} A_{k m}-A_{i l} x_{k}^{\prime} x_{m}^{\prime}-A_{k m} x_{i}^{\prime} x_{l}^{\prime}+x_{i}^{\prime} x_{k}^{\prime} x_{l}^{\prime} x_{m}^{\prime}\right)\right]$.

Now from the properties of the determinant $\left|a_{i k}\right|$, we have

$$
\sum_{i} a_{i k} A_{i l}=0 \quad(k \neq l) ; \quad \sum_{i} a_{i k} A_{i l}=1 \quad(k=l) .
$$

By using relations similar to (9) and also applying (2), (8) is easily reduced (after corresponding changes of subscripts) to

$$
\frac{1}{\rho^{2}}=\sum_{i} \frac{\partial L}{\partial x_{i}}\left[\sum_{l} \frac{\partial L}{\partial x_{l}}\left(A_{i l}-x_{i}^{\prime} x_{l}^{\prime}\right)\right] \text {. }
$$

Let us now consider the $\infty^{n-1}$ curves of the family which pass through a given point, $M_{0}$, of the space. Then since $\partial L / \partial x_{i}$ and $A_{i l}$ are functions of the coördinates of the point only, they may be regarded as constants here, and (10) expresses the law of variation of the geodesic curvature with respect to the direction, $x_{i}^{\prime}$, of the curve through the given point.

If in (10) we introduce the direction constants of the principal geodesic normal $\ddagger$

$$
\xi_{i}=\rho\left[x_{i}^{\prime \prime}+\sum_{\lambda \mu}\left\{\begin{array}{c}
\lambda_{\mu} \\
i
\end{array}\right\} x_{\lambda}^{\prime} x_{\mu}^{\prime}\right]=\rho\left[\sum_{l} \frac{\partial L}{\partial x_{l}}\left(A_{i l}-x_{i}^{\prime} x_{l}^{\prime}\right)\right],
$$

we get the law of the variation of the curvature expressed more simply by the characteristic relation

$$
\frac{1}{\rho}=\sum_{i} \frac{\partial L}{\partial x_{i}} \xi_{i}
$$

* The geodesic curvature, $1 / \rho$, at a point $M_{0}$ of a curve, $c$, in a curved $n$-space, is defined as follows. Draw the geodesic, $g$, (lying in the space) passing through the point in the same direction as the curve. Lay off on $c$ and $g$ equal infinitesimal arc lengths, $M_{0} M_{1}$ and $M_{0} M_{2}$ respectively, then $1 / \rho=\operatorname{limit} 2 M_{1} M_{2} /\left(M_{0} M_{1}\right)^{2}$. The limiting position of the direction $M_{1} M_{2}$ defines the direction of the principal geodesic normal at the point $M_{0}$. It is important to note that this definition coincides with that of the ordinary curvature of a curve lying in a euclidean space. Cf. BIANchI, ibid., p. 363.

† BinNCEI, ibid., p. 364.

$\ddagger$ Bianchi, ibid,, p. 364.

Trans. Am. Math. Soc. 6 
We may interpret (12) as follows: If we draw all the $\infty^{n-1}$ geodesics through a given point, $M_{0}$, and measuring from $M_{0}$ we lay off on each of these a distance $\rho$ determined by (12), (where $\xi$ is the direction of the geodesic) we shall determine a system of $\infty^{n-1}$ points contained in a $V_{n-1}$, which are the centers of the $\infty^{n-1}$ geodesic circles - circles of constant geodesic radial distance - corresponding to the $\infty^{n-1}$ curves of the natural family.

But we may get a much more fruitful interpretation of (12) as follows. Consider the euclidean space, $S_{n}$, tangent to our curved space, $V_{n}$, at the point $M_{0}$. Draw, in the $S_{n}$, the tangent (straight) line to the geodesic of $V_{n}$ passing out in the direction of the principal geodesic normal, $\xi$, and lay off on this tangent the radius of geodesic curvature determined by (12) (measuring from $M_{0}$ ). The extremity, $P$, will be the center of geodesic curvature of the corresponding curve of the natural family. Query: what is the locus of all such points, $P$, in $S_{n}$ for all curves of a natural family passing through $M_{0}$ ? To answer this query we proceed as follows. We can easily build up a parameter system in $V_{n}$, such that the parameter curves at the particular point, $M_{0}$, in question, shall be mutually orthogonal. Hence $a_{i l}=0(i \neq l), A_{i l}=0(i \neq l)$ for the point $M_{0}$. In $S_{n}$, take $M_{0}$ as the origin of coördinates and the tangent lines to the orthogonal parameter curves through $M_{0}$, as the rectangular system of coördinate axes. If $\omega_{i}$ is the angle between the principal geodesic normal, $\xi$, and the parameter curve $u_{i}$, then the rectangular coördinates of $P$ are

$$
y_{i}=\rho \cos \omega_{i}=\rho \sqrt{a_{i i}} \xi_{i},
$$

and introducing these coördinates in (12), we have

$$
\sum_{i} \frac{\frac{\partial L}{\partial x_{i}}}{\sqrt{a_{i i}}} y_{i}=1 .
$$

This, being a linear relation in the rectangular coördinates, $y_{i}$, evidently picks out a euclidean space of $n-1$ dimensions $\left(S_{n-1}\right)$ in our tangent euclidean space of $n$ dimensions $\left(S_{n}\right)$. With every point of our $V_{n}$, there is thus associated a euclidean $S_{n-1}$, which is the locus of the centers of geodesic curvature of the $\infty^{n-1}$ curves of a natural family of $V_{n}$ passing through this point. We thus have

Theorem 1. Property A. The locus of the centers of geodesic curvature of the curves of a natural family passing through a given point is a euclidean space of $(n-1)$ dimensions.

From (14) we see that, in the $S_{n}$, the direction

$$
\frac{\partial L}{\partial x_{i}} / \sqrt{a_{i i}}
$$

is normal to the $S_{n-1}$. Disregarding our special orthogonal system of parameter 
curves built up at $M_{0}$, this direction is, in general, given by

$$
\sqrt{a_{i i}}\left(\sum_{l} A_{i l} \frac{\partial L}{\partial x_{l}}\right)
$$

referred to the coördinate system in $S_{n}$, and by the direction constants

$$
\sum_{l} A_{i l} \frac{\partial L}{\partial x_{l}}
$$

referred to a general system of parameter curves in $V_{n}$.

In case our natural family represents a system of trajectories under conservative forces, this normal direction gives the direction of the force vector at the point. For, since the condition of orthogonality of the direction (15) and any arbitrary direction $d x_{i}$, viz.,

$$
\sum_{i k} a_{i k}\left(\sum_{l} A_{k l} \frac{\partial L}{\partial x_{l}}\right) d x_{i}=\sum_{l} \frac{\partial L}{\partial x_{l}} d x_{l}=0,
$$

is fulfilled for the equipotential hypersurface $L=$ constant, the force vector is evidently given by (15).

TheOREM 2. In the system of trajectories under conservative forces, the euclidean $S_{n-1}$ of theorem 1, associated with each point of $V_{n}$, is normal to the force vector through the point.

For any natural family, the direction (15) has another very important relation. Consider the pencil of $\left(\infty^{1}\right)$ directions through $M_{0}$,

$$
\begin{aligned}
& \zeta_{i}=\alpha x_{i}^{\prime}+\beta \frac{\xi_{i}}{\rho} \\
& (\alpha, \beta, \text { parameters ) } \\
& (i=1,2, \cdots, n)
\end{aligned}
$$

determined by the tangent direction and that of the principal geodesic normal to any curve of the system. The pencil of $\left(\infty^{1}\right)$ geodesics passing out in this pencil of directions form a two-dimensional spread, which is called the osculating geodesic surface to the chosen curve at $M_{0}$. Introducing in (17) the values of $\xi_{i} / \rho$ given by (11), we have

$$
\zeta_{i}=\alpha x_{i}^{\prime}+\beta \sum_{l} \frac{\partial L}{\partial x_{l}}\left(A_{i l}-x_{i}^{\prime} x_{l}^{\prime}\right)=x_{i}^{\prime}\left(\alpha-\beta \sum_{l} \frac{\partial L}{\partial x_{l}} x_{l}^{\prime}\right)+\beta \sum_{l} A_{i l} \frac{\partial L}{\partial x_{l}},
$$

and if we choose

$$
\frac{\alpha}{\beta}=\sum_{l} \frac{\partial L}{\partial x_{l}} x_{l}^{\prime},
$$

which is the same for all values of the subscript, $i$, we have

$$
\zeta_{i}=\beta \sum_{l} A_{i l} \frac{\partial L}{\partial x_{i}}
$$


this direction is independent of the direction in which we pass out from the point $M_{0}$ and hence is contained in all the osculating geodesic surfaces at the point; this direction coincides with the direction given by (15), normal to the $S_{n-1}$ of Theorem 1. Hence

Theorem 3. Property B. The osculating geodesic surfaces to the $\infty^{n-1}$ curves of a natural family passing through a common point of $V_{n}$ form a bundle of surfaces, i. e. they all contain a fixed direction (and hence the geodesic in that direction), which is normal to the euclidean $S_{n-1}$ defined by property $A$.

It is interesting to combine properties $A$ and $B$ into a single theorem. For this purpose, consider again the tangent space $S_{n}$ at $M_{0}$. Project the curves of a natural family in $V_{n}$, which pass through $M_{0}$, orthogonally upon $S_{n}$. The geodesic curvature, center of geodesic curvature and osculating geodesic surfaces of the curves of $V_{n}$ through $M_{0}$ coincide with the curvature, center of curvature and osculating planes of their projections in $S_{n}$ through $M_{0}$. And the properties of these projections may now be stated as

THEOREM 4. If the curves of a natural family, which pass through a common point of $V_{n}$, are projected orthogonally upon the tangent euclidean space $S_{n}$ at that point, the $\infty^{n-1}$ osculating circles of the projections will form an $(n-1)$ fold bundle, i. e., they will all have a second point in common.

\section{§3. Converses of properties $A$ and $B$.}

We have now shown that all natural families in a general curved space of $n$ dimensions possess properties $A$ and $B$. If we are to discover whether these properties are characteristic of natural families, we shall have to attack the converse problem, viz: Are all families of $\infty^{2(n-1)}$ curves in $a V_{n}$, which possess properties $A$ and $B$, natural families?

To answer this query, we may start by assuming the differential equations of any such system of curves in $V_{n}$ to be of the form

$$
x_{i}^{\prime \prime}=\chi_{i}\left(x_{1}, x_{2}, \cdots x_{n} ; x_{1}^{\prime}, x_{2}^{\prime}, \cdots x_{n}^{\prime}\right) \quad(i=1,2, \cdots, n) .
$$

Property $A$ says that the locus of centers of geodesic curvature of the $\infty^{n-1}$ curves passing through a point $M_{0}$ is a euclidean $S_{n-1}$. This, as we have seen, is characterized by an equation of the form (14), and for a general parameter system may be written,

$$
\sum_{i} \sqrt{a_{i i}}\left(\sum_{l} \phi_{l} A_{i l}\right) y_{i}=1,
$$

where $\phi_{1}, \phi_{2}, \cdots \phi_{n}$ are $n$ arbitrary point functions. Here, the $y_{i}$ are the orthogonal projections of the center of geodesic curvature on the tangent lines to the parameter curves through $M_{0}$ as origin, and hence are given by

$$
y_{i}=\rho \cos \omega_{i}=\frac{\rho}{\sqrt{a_{i i}}} \sum_{i} a_{i k} \xi_{k}
$$


Introducing these and the general values of $\rho$ and $\xi_{i}$ given by (7) and (11), . we get

$$
\begin{aligned}
\sum_{i k} a_{i k}\left[x_{i}^{\prime \prime}+\sum_{\lambda \mu}\left\{\begin{array}{c}
\lambda \mu \\
i
\end{array}\right\} x_{\lambda}^{\prime} x_{\mu}^{\prime}\right]\left[x_{k}^{\prime \prime}+\sum_{\lambda \mu}\left\{\begin{array}{c}
\lambda_{\mu} \\
k
\end{array}\right\} x_{\lambda}^{\prime} x_{\mu}^{\prime}\right] \\
=\sum_{i} \phi_{i}\left[x_{i}^{\prime \prime}+\sum_{\lambda \mu}\left\{\begin{array}{c}
\lambda \mu \\
i
\end{array}\right\} x_{\lambda}^{\prime} x_{\mu}^{\prime}\right] .
\end{aligned}
$$

Property $B$ says that the osculating geodesic surfaces at a point $M_{0}$ all pass through the normal to the $S_{n-1}$ of property $A$, i. e.

$$
\beta \sum_{l} \phi_{l} A_{i l}=\alpha x_{i}^{\prime}+\beta \frac{\xi_{i}}{\rho}=\alpha x_{i}^{\prime}+\beta\left[x_{i}^{\prime \prime}+\sum_{\lambda \mu}\left\{\begin{array}{c}
\lambda \mu \\
i
\end{array}\right\} x_{\lambda}^{\prime} x_{\mu}^{\prime}\right]
$$

From the $n$ equations (24), we have

$$
(i=1,2, \ldots, n) \text {. }
$$

$$
x_{i}^{\prime \prime}+\sum_{\lambda \mu}\left\{\begin{array}{c}
\lambda \mu \\
i
\end{array}\right\} x_{\lambda}^{\prime} x_{\mu}^{\prime}=\sum_{l} \phi_{l} A_{i l}-\frac{\alpha}{\beta} x_{i} \quad(i=1,2, \cdots, n) .
$$

Introducing these in $\left(22^{\prime}\right)$, we have

$$
\sum_{i k} a_{i k}\left[\sum_{l} \phi_{l} A_{i l}-\frac{\alpha}{\beta} x_{i}^{\prime}\right]\left[\sum_{l} \phi_{l} A_{k l}-\frac{\alpha}{\beta} x_{k}^{\prime}\right]=\sum_{i} \phi_{i}\left[\sum_{l} \phi_{l} A_{i l}-\frac{\alpha}{\beta} x_{i}^{\prime}\right]
$$

Expanding and bringing in the general relation (2) and (9), we reduce this easily to

$$
\left(\frac{\alpha}{\beta}\right)^{2}-\frac{\alpha}{\beta} \sum_{l} \phi_{l} x_{l}^{\prime}=0
$$

Disregarding the trivial solution $\alpha / \beta=0$, which causes the pencil of directions determining the osculating geodesic surfaces to degenerate to a single direction, we have finally

$$
\frac{\alpha}{\beta}=\sum_{l} \phi_{l} x_{l}^{\prime}
$$

and introducing this value in the equations (25), we have

$$
x_{i}^{\prime \prime}+\sum_{\lambda \mu}\left\{\begin{array}{c}
\lambda \mu \\
i
\end{array}\right\} x_{\lambda}^{\prime} x_{\mu}^{\prime}=\sum_{l} \phi_{l}\left(A_{i l}-x_{i}^{\prime} x_{l}^{\prime}\right) \quad(i=1,2, \ldots, n),
$$

together with equation (2) as the differential equations of the family of curves in $V_{n}$ possessing properties $A$ and $B$.

THEOREM 5. The differential equations of the most general system of curves in $V_{n}$ having properties $A$ and $B$ are given by equations (29), where $\phi_{1}, \phi_{2}, \cdots \phi_{n}$ are arbitrary point functions. The converse is valid also. 
Equations (29) are seen to include equations

$$
x_{i}^{\prime \prime}+\sum_{\lambda \mu}\left\{\begin{array}{c}
\lambda \mu \\
i
\end{array}\right\} x_{\lambda}^{\prime} x_{\mu}^{\prime}=\sum_{l} \frac{\partial L}{\partial x_{l}}\left(A_{i l}-x_{i}^{\prime} x_{l}^{\prime}\right) \quad(i=1,2, \cdots, n)
$$

as a special case. Thus (29) will represent a natural family, when and only when the $n$ arbitrary functions, $\phi_{i}$, are the partial derivatives with respect to $x_{i}$ of a single function, $L$, i. e. when the $\phi_{i}$ satisfy the system of equations

$$
\frac{\partial \phi_{l}}{\partial x_{i}}=\frac{\partial \phi_{i}}{\partial x_{l}} \quad\left(\begin{array}{l}
i=1,2, \ldots, n \\
l=1,2, \ldots, n
\end{array}\right) .
$$

Properties $A$ and $B$, therefore, do not characterize natural families; we must therefore seek additional properties. We shall refer to the systems defined by equations (29) as systems of type $(G)$, and those defined by equations (6) as systems of type $(N)$.

\section{§. Iyperosculating geodesic circles. - Property $C$.}

Let us find the conditions that the osculating geodesic surface at a point $M_{0}$ shall hyperosculate, i. e. have four-point contact with the curve.

Consider the pencil of directions

$$
\xi_{i}=\alpha \xi_{i}^{(1)}+\beta \xi_{i}^{(2)} \quad(i=1,2, \cdots, n)
$$

(which determine a two-dimensonal spread in $V_{n}$ ) determined by the fixed directions $\xi^{(!)}$and $\xi^{(2)}$ through the point $M_{0}$, which we shall take as origin of coördinates. The $\infty^{1}$ geodesics which pass through $M_{0}$, out along this pencil of directions, determine a geodesic surface $\sigma$. We may express the coördinates $x$ of a moving point on any one of these geodesics in any direction, say $\xi$, by means of an expansion in powers of the length of arc, $s$, measured from the point $M_{0}$, thus:

$$
x_{i}=x_{i}^{\prime} s+x_{i}^{\prime \prime} \frac{s^{2}}{2}+x_{i}^{\prime \prime \prime} \frac{s^{3}}{6}+\cdots
$$

Along a geodesic, we have *

(33) $x_{i}^{\prime \prime}=-\sum_{\lambda \mu}\left\{\begin{array}{c}\lambda \mu \\ i\end{array}\right\} x_{\lambda}^{\prime} x_{\mu}^{\prime}, \quad x_{i}^{\prime \prime \prime}=\sum_{\lambda \mu \nu}\left(2 \sum_{h}\left\{\begin{array}{c}\lambda h \\ i\end{array}\right\}\left\{\begin{array}{c}\mu \nu \\ h\end{array}\right\}-\frac{\partial}{\partial x_{\nu}}\left\{\begin{array}{c}\lambda \mu \\ i\end{array}\right\}\right) x_{\lambda}^{\prime} x_{\mu}^{\prime} x_{\nu}^{\prime}$

putting in the value of $\xi_{i}$, from (31), and further choosing upon the surface $\sigma$, the variable coördinates

$$
u_{1}=s \alpha, \quad u_{2}=s \beta,
$$

\footnotetext{
* Bianchi, ibid., p. 334.
} 
we may finally replace (32) by

$$
x_{i}=\xi_{i}^{(1)} u_{1}+\xi_{i}^{(2)} u_{2}-\frac{1}{2} \sum_{\lambda \mu}\left\{\begin{array}{c}
\lambda_{\mu} \\
i
\end{array}\right\}\left(\xi_{\lambda}^{(1)} u_{1}+\xi_{\lambda}^{(2)} u_{2}\right)\left(\xi_{\mu}^{(1)} u_{1}+\xi_{\mu}^{(2)} u_{2}\right)
$$

$+\frac{1}{6} \sum_{\lambda \mu \nu}\left(2 \sum_{h}\left\{\begin{array}{c}\lambda h \\ i\end{array}\right\}\left\{\begin{array}{c}\mu \nu \\ h\end{array}\right\}-\frac{\partial}{\partial x_{\nu}}\left\{\begin{array}{c}\lambda \mu \\ i\end{array}\right\}\right)\left(\xi_{\lambda}^{(1)} u_{1}+\xi_{\lambda}^{(2)} u_{2}\right)\left(\xi_{\mu}^{(1)} u_{1}+\xi_{\mu}^{(2)} u_{2}\right)\left(\xi_{\nu}^{(1)} u_{1}+\xi_{\nu}^{(2)} u_{2}\right)+\cdots$

We are now ready to develop the conditions for contact of different orders between our geodesic surface $\sigma$ and any curve whatever passing through $M_{0}$ in any direction $x^{\prime}$.

We shall employ the conditions for contact between a surface and a curve developed by Picard for a euclidean $S_{3}$, which can easily be shown to be extensible to a surface and a curve in any curved space.*

Applying these to our surface $\sigma$ and curve in direction $x^{\prime}$, we obtain for contact of the first order, the conditions

$$
\lambda_{1} \xi_{i}^{(1)}+\mu_{1} \xi_{i}^{(2)}-x_{i}^{\prime}=0
$$$$
(i=1,2, \ldots, n) \text {. }
$$

This is evidently satisfied if $\xi_{i}^{(1)}=x_{i}^{\prime}$, i. e. if $\sigma$ contains the tangent direction to the curve. From (35) we also have

$$
\lambda_{1}=\frac{1}{D}\left|\begin{array}{cc}
x_{\lambda}^{\prime} & \xi_{\lambda}^{(2)} \\
x_{\mu}^{\prime} & \xi_{\mu}^{(2)}
\end{array}\right|, \quad \mu_{1}=\frac{1}{D}\left|\begin{array}{cc}
\xi_{\lambda}^{(1)} & x_{\lambda}^{\prime} \\
\xi_{\mu}^{(1)} & x_{\mu}^{\prime}
\end{array}\right|, \quad D=\left|\begin{array}{cc}
\xi_{\lambda}^{(1)} & \xi_{\lambda}^{(2)} \\
\xi_{\mu}^{(1)} & \xi_{\mu}^{(2)}
\end{array}\right| .
$$

For contact of the second order (osculation), we get in addition to (35) the conditions

$$
\begin{array}{r}
\lambda_{2} \xi_{i}^{(1)}+\mu_{2} \xi_{i}^{(2)}-\frac{1}{2}\left[\sum_{\lambda \mu}\left\{\begin{array}{c}
\lambda \mu \\
i
\end{array}\right\}\left(\lambda_{1} \xi_{\lambda}^{(1)}+\mu_{1} \xi_{\lambda}^{(2)}\right)\left(\lambda_{1} \xi_{\mu}^{(1)}+\mu_{1} \xi_{\mu}^{(2)}\right)\right]-\frac{1}{2} x_{i}^{\prime \prime}=0 \\
(i=1,2, \ldots, n)
\end{array}
$$

and introducing here the values of $\lambda_{1}, \mu_{1}$ from (36), we have

$$
\lambda_{2} \xi_{i}^{(1)}+\mu_{2} \xi_{i}^{(2)}-\frac{1}{2}\left[x_{i}^{\prime \prime}+\sum_{\lambda \mu}\left\{\begin{array}{c}
\lambda_{\mu} \\
i
\end{array}\right\} x_{\lambda}^{\prime} x_{\mu}^{\prime}\right]=0 \quad(i=1,2, \ldots, n) .
$$

* PICARD, Traité d'analyse, vol. 1, chap. XII. Extending the conditions as given on p. 338, to any curve $x_{i}=f_{i}(t)(i=1,2, \ldots, n)$ and any surface $x_{i}=F_{i}(u, v)(i=1,2, \ldots, n)$, we have at any common point $x_{0}: t=t_{0}, u=u_{0}, v=v_{0}$, for contact of the

1st order:

$$
\lambda_{1} \frac{\partial F_{i}}{\partial u_{0}}+\mu_{1} \frac{\partial F_{i}}{\partial v_{0}}-f_{i}^{\prime}\left(t_{0}\right)=0 \quad(i=1,2, \cdots, n)
$$

2d order, also: $\quad \lambda_{2} \frac{\partial F_{i}}{\partial u_{0}}+\mu_{2} \frac{\partial F_{i}}{\partial v_{0}}+\frac{1}{2}\left(\lambda_{1} \frac{\partial}{\partial u_{0}}+\mu_{1} \frac{\partial}{\partial v_{0}}\right) F_{i}-\frac{1}{2} f_{i}^{\prime \prime}\left(t_{0}\right)=0 \quad(i=1,2, \cdots, n)$

3d order, also: $\lambda_{3} \frac{\partial F_{i}}{\partial u_{0}}+\mu_{0} \frac{\partial F_{i}}{\partial v_{0}}+\left(\lambda_{1} \frac{\partial}{\partial u_{0}}+\mu_{1} \frac{\partial}{\partial v_{0}}\right)\left(\lambda_{2} \frac{\partial}{\partial u_{0}}+\mu_{2} \frac{\partial}{\partial v_{0}}\right) F_{1}$

$$
+\frac{1}{6}\left(\lambda_{1} \frac{\partial}{\partial u_{0}}+\mu_{1} \frac{\partial}{\partial v_{0}}\right)^{3} F_{i}-\frac{1}{6} f_{i}^{\prime \prime \prime}\left(t_{0}\right)=0 \quad(i=1,2, \cdots, n)
$$

where $\lambda_{1}, \mu_{3} ; \lambda_{2}, \mu_{2} ; \lambda_{2}, \mu_{4}$ are arbitrary parameters. 
This is evidently satisfied if

$$
\xi_{i}^{(2)}=x_{i}^{\prime \prime}+\sum_{\lambda \mu}\left\{\begin{array}{c}
\lambda \mu \\
i
\end{array}\right\} x_{\lambda}^{\prime} x_{\mu}^{\prime} \quad(i=1,2, \ldots, n),
$$

i. e. if $\sigma$ contains the principal geodesic normal to the curve.

Our surface $\sigma$, represented by (34), will therefore have contact of the second order, i. e. will be the osculating geodesic surface to the curve, provided it contains the directions of the tangent line and the principal geodesic normal to the curve. We may therefore find the equation of the osculating geodesic surface by replacing in (34) the directions $\xi^{(1)}$ and $\xi^{(2)}$ by these latter directions.

For contact of the third order (hyperosculation), we must have, in addition to (35) and (37), the conditions

$$
\begin{aligned}
\lambda_{3} \xi_{i}^{(1)} & +\mu_{3} \xi_{i}^{(2)}-\frac{1}{2} \sum_{\lambda \mu}\left\{\begin{array}{c}
\lambda \mu \\
i
\end{array}\right\}\left[\left(\lambda_{1} \xi_{\lambda}^{(1)}+\mu_{1} \xi_{\lambda}^{(2)}\right)\left(\lambda_{2} \xi_{\mu}^{(1)}+\mu_{2} \xi_{\mu}^{(2)}\right)\right. \\
& \left.+\left(\lambda_{1} \xi_{\mu}^{(1)}+\mu_{1} \xi_{\mu}^{(2)}\right)\left(\lambda_{2} \xi_{\lambda}^{(1)}+\mu_{2} \xi_{\lambda}^{(2)}\right)\right]+\frac{1}{6} \sum_{\lambda \mu \nu}\left[2 \sum_{h}\left\{\begin{array}{c}
\lambda h \\
i
\end{array}\right\}\left\{\begin{array}{c}
\mu \nu \\
h
\end{array}\right\}\right. \\
& \left.-\frac{\partial}{\partial x_{\nu}}\left\{\begin{array}{c}
\lambda \mu \\
i
\end{array}\right\}\right]\left[\left(\lambda_{1} \xi_{\mu}^{(1)}+\mu_{1} \xi_{\lambda}^{(2)}\right)\left(\lambda_{1} \xi_{\mu}^{(1)}+\mu_{1} \xi_{\mu}^{(2)}\right)\left(\lambda_{1} \xi_{\nu}^{(1)}+\mu_{1} \xi_{\nu}^{(2)}\right)\right]-\frac{1}{6} x_{i}^{\prime \prime \prime}=0,
\end{aligned}
$$

where $i=1,2, \cdots, n$; and introducing the values of $\lambda_{1}, \mu_{1}$ from (36) and values. for $\lambda_{2}, \mu_{2}$ gotten similarly from (37'), we reduce (39) to

$$
\begin{aligned}
& \lambda_{3} \xi_{i}^{(1)}+\mu_{3} \xi_{i}^{(2)}-\frac{1}{2} \sum_{\lambda \mu}\left\{\begin{array}{c}
\lambda \mu \\
i
\end{array}\right\} x_{\lambda}^{\prime}\left(x_{\mu}^{\prime \prime}+\sum_{\nu \tau}\left\{\begin{array}{c}
\nu \tau \\
\mu
\end{array}\right\} x_{\nu}^{\prime} x_{\tau}^{\prime}\right) \\
& +\frac{1}{6} \sum_{\lambda \mu \nu}\left(2 \sum_{h}\left\{\begin{array}{c}
\lambda h \\
i
\end{array}\right\}\left\{\begin{array}{c}
\mu \nu \\
h
\end{array}\right\}-\frac{\partial}{\partial x_{\nu}}\left\{\begin{array}{c}
\lambda \mu \\
i
\end{array}\right\}\right) x_{\lambda}^{\prime} x_{\mu}^{\prime} x_{\nu}^{\prime}-\frac{1}{6} x_{i}^{\prime \prime \prime}=0(i=1,2, \ldots, n) .
\end{aligned}
$$

Finally combining (35), $\left(37^{\prime}\right)$ and $\left(39^{\prime}\right)$, we have, as the conditions that the geodesic surface $\sigma$ should have contact of the third order with the curve, in other words, that the osculating geodesic surface to the curve should hyperosculate,

$$
\begin{aligned}
\lambda_{3} x_{i}^{\prime}+\mu_{3}\left[x_{i}^{\prime \prime}\right. & \left.+\sum_{\lambda \mu}\left\{\begin{array}{c}
\lambda_{\mu} \\
i
\end{array}\right\} x_{\lambda}^{\prime} x_{\mu}^{\prime}\right]-\frac{1}{2} \sum_{\lambda \mu}\left\{\begin{array}{c}
\lambda_{\mu} \\
i
\end{array}\right\} x_{\lambda}^{\prime}\left(x_{\mu}^{\prime \prime}+\sum_{\nu \tau}\left\{\begin{array}{c}
\nu \tau \\
\mu
\end{array}\right\} x_{\nu}^{\prime} x_{\tau}^{\prime}\right) . \\
& +\frac{1}{6} \frac{d}{d s}\left[x_{i}^{\prime \prime}+\sum_{\lambda \mu}\left\{\begin{array}{c}
\lambda \mu \\
i
\end{array}\right\} x_{\lambda}^{\prime} x_{\mu}^{\prime}\right]-\frac{1}{6} x_{i}^{\prime \prime \prime}=0 \quad(i=1,2, \ldots, n) .
\end{aligned}
$$

Applying (40) to the system $(G)$ defined by

$$
x_{i}^{\prime \prime}+\sum_{\lambda \mu}\left(\begin{array}{c}
\lambda \mu \\
i
\end{array}\right) x_{\lambda}^{\prime} x_{\mu}^{\prime}=\sum_{l} \phi_{l}\left(A_{i l}-x_{i}^{\prime} x_{l}^{\prime}\right) \quad(i=1,2, \ldots, n)
$$


and introducing $B_{i}$ as an abbreviation for the right member of (29), we obtain

$$
\lambda_{3} x_{i}^{\prime}+\mu_{3} B_{i}-\frac{1}{6} \sum_{\lambda \mu}\left\{\begin{array}{c}
\lambda \mu \\
i
\end{array}\right\} x_{\lambda}^{\prime} B_{\mu}-\frac{1}{6} B_{i}^{\prime}=0 \quad(i=1,2, \ldots, n) .
$$

If from these $n$ equations we eliminate the parameters $\lambda_{3}, \mu_{3}$ and introduce the values of $B_{i}, B_{i}^{\prime}$, we get, after considerable reduction,

$$
\left|\begin{array}{lll}
x_{1}^{\prime} & \sum_{l} \phi_{l} A_{1 l} & \sum_{l} \phi_{l}\left(\sum_{\lambda \mu}\left\{\begin{array}{c}
\lambda_{\mu} \\
1
\end{array}\right\} A_{\mu l} x_{\lambda}^{\prime}\right)+\sum_{l}\left(\phi_{l} A_{1 l}\right)^{\prime} \\
x_{2}^{\prime} & \sum_{l} \phi_{l} A_{2 l} & \sum_{l} \phi_{l}\left(\sum_{\lambda \mu}\left\{\begin{array}{c}
\lambda_{\mu} \\
2
\end{array}\right\} A_{\mu l} x_{\lambda}^{\prime}\right)+\sum_{l}\left(\phi_{l} A_{2 l}\right)^{\prime} \\
x_{k}^{\prime} & \sum_{l} \phi_{l} A_{k l} & \sum_{l} \phi_{l}\left(\sum_{\lambda \mu}\left\{\begin{array}{c}
\lambda_{\mu} \\
k
\end{array}\right\} A_{\mu l} x_{\lambda}^{\prime}\right)+\sum_{l}\left(\phi_{l} A_{k l}\right)^{\prime}
\end{array}\right|=0(k=3,4,5, \ldots, n) .
$$

We may then state that the $(n-2)$ equations, $(42)$, are the conditions that the osculating geodesic surface at a point $M_{0}$ of any curve of the type $(G)$ should hyperosculate that curve.

These $(n-2)$ equations evidently do not pick out a finite number of directions through $M_{0}$ in which the hyperosculation property holds. Studying equations (42) we may simplify their form by choosing the $x_{1}$ parameter curve, at $M_{0}$, in the fixed direction $\sum_{l} \phi_{l} A_{i l}(i=1,2, \cdots n)$; then $\sum_{l} \phi_{l} A_{i l}=0(i \neq 1)$, and

(42) becomes

$$
\frac{\left(\sum_{l} \phi_{l} A_{1 l}\right) \sum_{\lambda}\left\{\begin{array}{c}
\lambda 1 \\
2
\end{array}\right\} x_{\lambda}^{\prime}+\sum_{l}\left(\phi_{l} A_{2 l}\right)^{\prime}}{x_{2}^{\prime}}=\frac{\left(\sum_{l} \phi_{l} A_{1 l}\right) \sum_{\lambda}\left\{\begin{array}{c}
\lambda 1 \\
k
\end{array}\right\} x_{\lambda}^{\prime}+\sum_{l}\left(\phi_{l} A_{k l}\right)^{\prime}}{x_{k}^{\prime}}
$$

We note here that the term containing $x_{1}^{\prime}$ in the denominator is missing to complete the symmetry, and it will be our next effort to supply such a term.

Consider a point $M_{0}$ of a curve $(c)$, of the general type $(G)$, and draw in its osculating geodesic surface, $\sigma$, at that point, the geodesic circle, $\gamma$, (curve of constant geodesic curvature) which osculates the curve $c$. We should note that at a point of $c$, and lying in $\sigma$, there can be drawn one and only one such curve $\gamma$. We shall term $\gamma$ the osculating geodesic circle of $c$ at $M_{0}$. Immediately the question arises: when urill $\gamma$ hyperosculate $c$ ? Evidently we need merely add to the conditions (43), that $\sigma$ should hyperosculate $c$, the following necessary and sufficient condition

$$
\frac{d \rho}{d s}=0
$$

For the curve of type $(G)$ we have

$$
\frac{1}{\rho^{2}}=\sum_{i} \phi_{i}\left[\sum_{l} \phi_{l}\left(A_{i l}-x_{i}^{\prime} x_{l}^{\prime}\right)\right] .
$$


Applying (44) to this, and using (29) and the above choice of the $x_{1}$ parameter curve, we get after some reduction,

$$
\begin{aligned}
2 \phi_{1}^{\prime}\left(\sum_{l} \phi_{l} A_{1 l}\right)+\sum_{i l} \phi_{i} \phi_{l} A_{i l}^{\prime}-2 \sum_{i} \phi_{i} x_{i}^{\prime}\left[\sum_{l} \phi_{l}^{\prime} x_{l}^{\prime}+\phi_{1}\left(\sum_{l} \phi_{l} A_{1 l}\right)\right. \\
\left.-\left(\sum_{l} \phi_{l} x_{l}^{\prime}\right)^{2}-\left(\sum_{l} \phi_{l} A_{1 l}\right) \sum_{\lambda \mu}\left[\begin{array}{c}
\lambda \mu \\
1
\end{array}\right] x_{\lambda}^{\prime} x_{\mu}^{\prime}\right]=0 .
\end{aligned}
$$

We may now state: the necessary and sufficient conditions that the osculating geodesic circle at a point of a curve of type $(G)$ should there hyperosculate the curve are that equations (43) and (46) be satisfied.

For a simplification of (46) we are driven to choose our parameter system in a more special form without thereby losing in generality. We shall choose a system of geodesic parameters. ${ }^{*}$ Then the element of arc length takes the form

$$
d s^{2}=d x_{1}^{2}+\sum_{i, k}^{2, \ldots n} a_{i k} d x_{i} d x_{k}
$$

and as we may still choose that particular parameter curve, $x_{1}$, which passes through the particular point $M_{0}$ under consideration, in the fixed direction $\sum_{l} \phi_{l} A_{i l}(i=1,2, \cdots n)$ at $M_{0}$, then by virtue of (47), we have

$$
\phi_{i}=0
$$

Under these hypotheses, (43) and (46) respectively reduce to

$$
\frac{\phi_{1} \sum_{\lambda}\left\{\begin{array}{c}
\lambda 1 \\
2
\end{array}\right\} x_{\lambda}^{\prime}+\sum_{l} A_{2 l} \phi_{l}^{\prime}}{x_{2}^{\prime}}=\frac{\phi_{1} \sum_{\lambda}\left\{\begin{array}{c}
\lambda 1 \\
k
\end{array}\right\} x_{\lambda}^{\prime}+\sum_{l} A_{k l} \phi_{l}^{\prime}}{x_{k}^{\prime}}=C \quad(k=3,4,5, \ldots, n) .
$$

(where $C$ is used merely as an abbreviation), and

$$
\phi_{1}^{\prime}-x_{1}^{\prime}\left[\sum_{l} \phi_{l}^{\prime} x_{l}^{\prime}+\phi_{1}^{2}-\phi_{1}^{2} x_{1}^{\prime 2}+\frac{1}{2} \phi_{1} \sum_{\lambda \mu} \frac{\partial a_{\lambda \mu}}{\partial x_{l}} x_{\lambda}^{\prime} x_{\mu}^{\prime}\right]=0 .
$$

Solving $\left(43^{\prime}\right)$ for $\phi_{l}^{\prime}$ and substituting in $\left(46^{\prime}\right)$ we find after considerable but simple reductions, and after casting out the trivial solution $1-x_{1}^{\prime 2}=0$,

$$
\frac{\phi_{1}^{\prime}}{x_{1}^{\prime}}-\phi_{1}^{2}=C \text {. }
$$

We finally have, combining $\left(43^{\prime}\right)$ and $\left(46^{\prime \prime}\right)$,

\footnotetext{
* Let the $x_{1}$ parameter curves be geodesics; further let the parameter $x_{1}$ represent the length of curve measured from the corresponding intersection with a definite orthogonal parameter hypersurface of the system $x_{1}=$ constant, e. g. $x_{1}=0$, so that the other hypersurfaces of the system are the loci of extremities of equal arcs. BinNCHI, ibid., pp. 336-337.
} 


$$
\frac{\sum_{\lambda} \frac{\partial \phi_{1}}{\partial x_{\lambda}} x_{\lambda}^{\prime}}{x_{1}^{\prime}}-\phi_{1}^{2}=\frac{\sum_{\lambda}\left[\phi_{1}\left\{\begin{array}{c}
\lambda 1 \\
k
\end{array}\right\}+\sum_{l} A_{k l} \frac{\partial \phi_{l}}{\partial x_{\lambda}}\right]^{x_{\lambda}^{\prime}}}{x_{k}^{\prime}}=C \quad(k=2,3, \ldots, n)
$$

as the conditions that the osculating geodesic circle at a point of a curve of type $(G)$ should there hyperosculate the curve.

We may write (48) in the more symmetric and abbreviated form

$$
\sum_{\lambda}[\lambda, k] \eta_{\lambda}=C \eta_{k}+\phi_{k}^{2} \eta_{k} \quad(k=1,2, \ldots, n),
$$

where

$$
[\lambda, k] \equiv\left[\phi_{1}\left\{\begin{array}{c}
\lambda 1 \\
k
\end{array}\right\}+\sum_{l} A_{k l} \frac{\partial \phi_{l}}{\partial x_{\lambda}}\right] ; \quad \eta_{k} \equiv x_{k}^{\prime}
$$

and under our special choice of the parameter system,

$$
\phi_{k}=0(k \neq 1) ; \quad[\lambda, 1]=\frac{\partial \phi_{l}}{\partial x_{1}} .
$$

The $n$ equations (48') give us in general $n$ distinct values for $C$, say

$$
C^{(a)}, C^{(\beta)}, \cdots,
$$

and introducing these in $\left(48^{\prime}\right)$ again, we have $n$ linear equations in $\eta_{i}$, which taken with

$$
\sum_{i k} a_{i k} \eta_{i} \eta_{k}=1
$$

give us, in general, $n$ sets of values of $\eta_{i}$, say

$$
\eta_{i}^{(1)}, \eta_{i}^{(2)}, \cdots \eta_{i}^{(n)} \quad(i=1,2, \ldots, n) .
$$

Equations (48') thus determine in general $n$ distinct directions through the point in which the corresponding osculating geodesic circles hyperosculate. We thus have

TheоReм 6. There are, in general, $n$ distinct directions through a point of $V_{n}$, in which the osculating geodesic circles hyperosculate the curves of a system of type $(G)$ passing out in these directions.

This property is evidently a consequence of properties $A$ and $B$. If we now pass from the curves of type $(G)$ to the special type $(N)$, we must introduce here the relations

$$
\frac{\partial \phi_{l}}{\partial x_{i}}=\frac{\partial \phi_{i}}{\partial x_{l}} \quad\left(\begin{array}{l}
i=1,2, \ldots, n \\
l=1,2, \ldots, n
\end{array}\right)
$$

The question arises: What effect do the relations (30) have upon the $n$ directions of theorem 6? To answer this, let us write down the conditions that these $n$ directions should be mutually orthogonal. Let $C^{(a)}$ and $C^{(\beta)}$ be any two distinct values of $C$, and let $\eta_{i}^{(a)}$ and $\eta_{m}^{(\beta)}$ be any two corresponding directions, i. e. sets of 
values of $\eta$. The condition of orthogonality for these two directions is given by

$$
\sum_{i m} a_{i m} \eta_{i}^{(\alpha)} \eta_{m}^{(\beta)}=0
$$

where we should remember that under our choice of geodesic parameters

$$
a_{1 l}=0 \quad(l \neq 1) \quad \text { and } \quad a_{11}=1 .
$$

Now from $\left(48^{\prime}\right)$ we have

$$
\begin{gathered}
\sum_{\lambda}[\lambda, i] \eta_{\lambda}^{(a)}=C^{(a)} \eta_{i}^{(a)}+\phi_{i}^{2} \eta_{i}^{(a)}, \\
\sum_{\nu}[\nu, m] \eta_{\nu}^{(\beta)}=C^{(\beta)} \eta_{m}^{(\beta)}+\phi_{m}^{2} \eta_{m}^{(\beta)} .
\end{gathered}
$$

Multiplying the first by $a_{i \nu} \eta_{\nu}^{(\beta)}$ and summing with respect to $i$ and $\nu$, and the second by $a_{m \lambda} \eta_{\lambda}^{(a)}$ and summing with respect to $m$ and $\lambda$, we obtain

$$
\begin{aligned}
\sum_{i \lambda \nu} a_{i \nu}[\lambda, i] \eta_{\lambda}^{(\alpha)} \eta_{\nu}^{(\beta)} & =C^{(\alpha)} \sum_{i \nu} a_{i \nu} \eta_{i}^{(\alpha)} \eta_{\nu}^{(\beta)}+\sum_{i \nu} a_{i \nu} \phi_{i}^{2} \eta_{i}^{(\alpha)} \eta_{\nu}^{(\beta)}, \\
\sum_{m \lambda \nu} a_{m \lambda}[\nu, m] \eta_{\nu}^{(\beta)} \eta_{\lambda}^{(\alpha)} & =C^{(\beta)} \sum_{m \lambda} a_{m \lambda} \eta_{m}^{(\beta)} \eta_{\lambda}^{(\alpha)}+\sum_{m \lambda} a_{m \lambda} \phi_{m}^{2} \eta_{m}^{(\beta)} \eta_{\lambda}^{(\alpha)} ;
\end{aligned}
$$

and since $\phi_{k}=0(\kappa \neq 1)$ and $a_{1 l}=0(l \neq 1), a_{11}=1$, the second terms in the left members of these equations each reduce to $\phi_{1}^{2} \eta_{1}^{(a)} \eta_{1}^{(\beta)}$. Hence, subtracting the two equations, we have

$$
\left[C^{(a)}-C^{(\beta)}\right] \sum_{i \nu} a_{i \nu} \eta_{i}^{(a)} \eta_{\nu}^{(\beta)}=\sum_{\nu \lambda}\left(\sum_{i} a_{i \nu}[\lambda, i]-\sum_{m} a_{m \lambda}[\nu, m]\right) \eta_{\lambda}^{(\alpha)} \eta_{\nu}^{(\beta)}
$$

Using the value of $[\lambda, i]$ defined by $(49)$, we can easily show that

$$
\sum_{i} a_{i \nu}[\lambda, i]=\frac{\partial \phi_{\nu}}{\partial x_{\lambda}}+\frac{1}{2} \phi_{1} \frac{\partial a_{\lambda \nu}}{\partial x_{1}} ; \sum_{m} a_{m \lambda}[\nu, m]=\frac{\partial \phi_{\lambda}}{\partial x_{\nu}}+\frac{1}{2} \phi_{1} \frac{\partial a_{\nu \lambda}}{\partial x_{1}},
$$

and hence (54) takes the form

$$
\left[C^{(\alpha)}-C^{(\beta)}\right] \sum_{i \nu} a_{i \nu} \eta_{i}^{(a)} \eta_{i}^{(\beta)}=\sum_{\nu \lambda}\left(\frac{\partial \phi_{\nu}}{\partial x_{\lambda}}-\frac{\partial \phi_{\lambda}}{\partial x_{\nu}}\right) \eta_{\lambda}^{(\alpha)} \eta_{\nu}^{(\beta)}
$$

Now as $C^{(\alpha)}$ and $C^{(\beta)}$ are distinct values of $C$, it is ovident that for the mutual orthogonality of our $n$ directions through a point, we have the necessary and sufficient conditions

$$
\frac{\partial \phi_{\nu}}{\partial x_{\lambda}}=\frac{\partial \phi_{\lambda}}{\partial x_{v}}
$$

where $\lambda, \nu$ range over the values from 1 to $n$. But these relations (56) are exactly our conditions (30) which transform the general type $(G)$ into the special type $(N)$. We thus have 
Theorem 7. Property C. For any natural family of curves in $V_{n}$, the $n$ directions at each point in which the osculating geodesic circles hyperosculate the curves passing out in these directions are mutually orthogonal.

Properties $A, B$ and $C$ are thus the characteristic properties of any natural family in any curved space of $n$ dimensions. In order, then, that any $\infty^{2(n-1)}$ curves in such a space should be the extremals connected with a variation problem of type

$$
\int F d s=\text { minimum, }
$$

they must possess these three properties. Our problem is therefore solved.

§5. Two-dimensional results. - Isogonal trajectories.

The case $n=2$ (an ordinary surface) is of special interest. In this case, if we choose our element of arc length in the isothermal form

$$
d s^{2}=\lambda(u, v)\left(d u^{2}+d v^{2}\right),
$$

we may write (1) in the form

$$
\int F(u, v) v^{\prime} \overline{\lambda\left(1+{v^{\prime 2}}^{2}\right.} d u=\text { minimum.* }
$$

The equation of the general type $(G)$ becomes

$$
v^{\prime \prime}=\left(\psi-\phi v^{\prime}\right)\left(1+{v^{\prime 2}}^{2},\right.
$$

where $\phi, \psi$ are any two arbitrary functions of $u$ and $v$. The equation of a natural family $(N)$ is

where

$$
v^{\prime \prime}=\left(L_{v}-L_{u} v^{\prime}\right)\left(1+{v^{\prime 2}}^{2},\right.
$$

$$
L=\log (F \sqrt{\lambda})
$$

and corresponds to the restriction

$$
\psi_{u}-\phi_{v}=0
$$

In this case, property $B$ falls away, as the osculating geodesic surfaces at a point all coincide with the surface itself. We may thus state the result:

Theorem 8. Any family of $\infty^{2}$ curves on a surface will be a natural family, when and only when the $\infty^{1}$ curves (one in each direction) passing through a point have the properties: (1) the locus of the centers of geodesic curvature is a straight line (or, the circles of curvature of the orthogonal projections, in the tangent plane, of the curves through a point have a second point in common); (2) the two directions in which the osculating geodesic circles hyperosculate the corresponding curves in those directions are orthogonal.

* Throughout this section, primes refer to total derivatives with respect to $u$, and subscripts to partial derivatives with respect to the indicated letter. 
The first of these properties is characteristic of the general type $(G)$ defined by (58).

There is another special case of (53) which is of noteworthy interest here; viz., the system of $\infty^{2}$ isogonal trajectories of a given set of $\infty^{1}$ curves on a surface. Let the given set be defined by the equation

$$
v^{\prime}=\alpha(u, v) \text {. }
$$

The equation of the isogonal trajectories is then easily found to be

$$
v^{\prime \prime}=\left(M_{u}+M_{v} v^{\prime}\right)\left(1+{v^{\prime 2}}^{2}\right)
$$

where

$$
M=\tan ^{-1} \alpha .
$$

This is evidently a special type of (58) corresponding to the restriction

$$
\psi_{v}+\phi_{u}=0
$$

We shall call this type, the type $(I)$.

THEOREM 9. For the system of isogonal trajectories on a surface, the locus of the centers of geodesic curvature of the curves passing through a point is a straight line.

To get a complete characterization, we shall show how the two types $(N)$ and (I) may be interchanged. Consider the transformation

$$
u_{1}=u, \quad v_{1}=v, \quad v_{1}^{\prime}=-\frac{1}{v^{\prime}}, \quad v_{1}^{\prime \prime}=-\frac{v^{\prime \prime}}{v^{\prime 3}} .
$$

Applying this to the general type (58), we find that the new differential equation is of the same form, with

$$
\phi_{1}=\psi, \quad \psi_{1}=-\phi .
$$

Hence if the original family is isogonal, the new family is natural and vice versa. We thus have

Theorem 10. The isogonal type

$$
v^{\prime \prime}=\left(\Omega_{u}+\Omega_{v} v^{\prime}\right)\left(1+{v^{\prime}}^{2}\right),
$$

which consists of all the isogonals of the simple system

$$
v^{\prime}=\tan \Omega,
$$

is converted by the transformation $(\Gamma)$ into the natural type

$$
v^{\prime \prime}=\left(\Omega_{v}-\Omega_{u} v^{\prime}\right)\left(1+{v^{\prime}}^{2}\right) .
$$

Thus with each system of the isogonal type there is associated by $(\Gamma)$ a definite family of the natural type, and vice versa; and properties of one give indirectly properties of the other, thus the associated system of a system of type $(I)$ will 
possess the property that through any point there are two directions in which the osculating geodesic circles hyperosculate.

The transformation $(\Gamma)$ is not a contact transformation. It defines the operation by means of which any geodesic curvature element $\left(u, v, v^{\prime}, v^{\prime \prime}\right)$ is transformed into another geodesic curvature element $\left(u, v,-1 / v^{\prime},-v^{\prime \prime} / v^{3}\right)$, by rotating it about its own point $(u, v)$ through a right angle-but the geodesic curvature is not preserved.* In the expression for geodesic curvature, it replaces $\lambda_{v}$ by $\lambda_{u}$ and $\lambda_{u}$ by $-\lambda_{v}$.

The transformation $\left(\Gamma^{2}\right)$ converts each geodesic curvature element into its opposite $\left(u, v, v^{\prime},-v^{\prime \prime}\right)$ and thus leaves each of the types invariant.

If we restrict the arbitrary functions $\psi$ and $\phi$ to both conditions

$$
\psi_{u}-\phi_{v}=0, \psi_{v}+\phi_{u}=0
$$

our system evidently consists of the isogonals of an isothermal system. $\dagger$ Thus we have

Theorem 11. The isogonals of an isothermal system form a natural family. The transformation $(\Gamma)$ converts such a system into one of the same sort.

Massachusetrs Institute of Technology, Boston, Mass.

* Compare this with the transformation in the plane, obtained by KASNER, ibid., p. 218.

$\dagger$ These are the conditions that $\phi=$ const. and $\psi=$ const. should form an isothermal system on the surface. Cf. BIANCHI, ibid., p. 98. 